\title{
INTRODUCTION \\ Entre Algérie et France : \\ Écrire une histoire sociale des Algériens au vingtième siècle
}

\author{
Muriel Cohen
}

Centre d'histoire sociale du XXe siècle - Paris 1

Annick Lacroix

Institut des sciences sociales du politique - ENS Cachan

L'histoire de l'Algérie colonisée a connu un grand renouveau depuis le début des années $2000 .{ }^{*}$ D'abord centrés sur la guerre d'indépendance, ces travauxqui ont privilégié une approche politique—ont progressivement renoué avec l'analyse de la période coloniale dans son ensemble ${ }^{1}$. De la même façon, plusieurs études ont porté sur l'encadrement et le contrôle de l'immigration algérienne en métropole entre 1954 et 1962 d'abord, puis sur une période plus large ${ }^{2}$. Ce dossier s'inscrit dans un mouvement ${ }^{3}$ qui cherche à présent à dépasser une histoire politique—souvent focalisée sur la répression-qui rigidifie le face à face entre colonisateurs et colonisés. Il ne s'agit aucunement de minorer la force de ce clivage, mais d'affiner l'analyse au-delà d'une stricte opposition binaire pour saisir d'autres logiques de structuration du social et faire émerger des groupes fondés sur des appartenances autres que juridiques et/ou raciales.

Le choix de se focaliser sur les Algériens se justifie largement par leur faible présence dans les travaux, au regard de ceux consacrés à la minorité européenne, il est vrai mieux représentée dans les sources. Une histoire des Algériens qui parte des pratiques des populations et de la vie quotidienne pour analyser en profondeur les structures sociales reste encore à écrire, y compris pour la métropole. Une histoire des Algériens à l'époque coloniale, mais qui ne soit pas nécessairement en lien avec le colonisateur, qui "réévalu[e] les domaines d'actions parfois autonomes des sujets coloniaux », largement 
minorés dans la plupart des travaux ${ }^{4}$. Une histoire qui questionnerait les intersections entre appartenances de classe, rapports de genre, oppositions entre rural et urbain, pratiques religieuses et statuts juridiques. Une histoire enfin qui explorerait les espaces de négociation et les formes de contournement que les acteurs se ménagent sans cesse, y compris dans le cadre asymétrique de la situation coloniale. Cette démarche n'est pas étrangère à celle du premier âge des Subaltern studies, qui avait cherché à mettre en avant la capacité d'action (agency) des dominés et leurs marges de manœuvre. Tout en prêtant attention aux cadres politiques et sociaux, les parcours individuels et les motivations des acteurs restent en effet des éléments essentiels de l'approche des sciences sociales.

L'entrée par le social ouvre en outre la possibilité d'une histoire à cheval entre Algérie et métropole, et entre périodes coloniale et post-coloniale, comme le préconisent Frederick Cooper et Ann Laura Stoler ${ }^{5}$. Elle permet de désenclaver l'histoire de l'Algérie coloniale en dépassant les frontières politiques, du point de vue à la fois spatial et temporel ${ }^{6}$. L'étude des administrations invite par exemple à penser d'un seul tenant l'ensemble métropole-colonie. Des services largement inspirés des normes et des pratiques en vigueur dans les administrations métropolitaines sont mis sur pied dans les départements algériens, comme c'est le cas des postes, télégraphes et téléphones (Annick Lacroix). Même si les autorités françaises font ensuite subir à ce modèle de nombreux ajustements justifiés par les supposées spécificités du terrain colonial. La création, en 1904, d'un corps des auxiliaires médicaux « indigènes » en est une illustration (Hannah-Louise Clark). Reste qu'au-delà de l'échelle politique et administrative imposée par la métropole, l'histoire des Algériens prend également sens à l'échelle du Maghreb, du monde arabe ou musulman. Les études qui suivent invitent également à rompre avec une histoire de l'Algérie qui a pu faire de l'épilogue guerrier sa principale clé d'interprétation. Elles éclairent la période charnière de l'entre-deux-guerres en Algérie (Annick Lacroix et HannahLouise Clark), apportent un regard décalé sur la guerre d'indépendance (Neil Macmaster et Emmanuel Blanchard) et ne se limitent pas par principe à la rupture politique de 1962 qui, par exemple, n'a pas d'effet immédiat sur le sort des migrants algériens (Muriel Cohen).

\section{Sujets, musulmans, indigènes ou Algériens ?}

Au-delà des déclarations d'intention, écrire l'histoire sociale des Algériens nécessite des sources et des outils spécifiques. L'exploration des groupes sociaux suppose également une réflexion sur le choix du vocabulaire. En Algérie, le statut juridique de la population colonisée ne cesse de se transformer, même après le sénatus-consulte du 14 juillet 1865 qui fixe la condition des "indigènes ", Français de nationalité mais non citoyens. Les termes $\mathrm{d}^{\prime}$ « indigènes ", "sujets français », " musulmans » rivalisent avec celui 
d'«Algériens ». Ce «malaise dans les catégories » ${ }^{7}$ invite à historiciser les dénominations mobilisées par l'administration coloniale et par les acteurs euxmêmes. Le chercheur peut-il, comme le font les sources qu'il utilise, assigner la population colonisée à une religion ? Peut-il utiliser le terme indigène et la mise à distance par des guillemets suffit-elle à souligner la violence symbolique inhérente aux catégories promues par le droit colonial ? La difficulté consiste à employer un vocabulaire rigoureux, tout en évitant les qualificatifs offensants ${ }^{8}$. Périphrases et expressions composées se révèlent alors souvent nécessaires.

On parlera donc, dans les articles qui suivent, de populations colonisées, d'employés de l'État non-citoyens mais aussi de migrants, de villageois ou de militants algériens. Le terme "algérien » est en partie anachronique-voire téléologique-pour le début du vingtième siècle, à une époque où il est revendiqué par les Européens installés durablement sur place. Il se répand pourtant après 1945 pour désigner les migrants venus en métropole ${ }^{9}$, à côté des expressions "Français musulmans d'Algérie » ou " Nord-africains », qui restent plus courantes. Algériens est surtout la catégorie nationale promue par les militants indépendantistes, et leur victoire explique la diffusion du terme dans l'historiographie. On perçoit ici combien ces enjeux de dénomination revêtent aussi une dimension politique. L'indépendance redéfinit plus fermement le clivage entre Algériens et Français, sans mettre fin aux circulations entre les deux côtés de la Méditerranée, aux unions mixtes, ni aux situations juridiques ambiguës.

\section{Un champ historiographique en friche}

L'histoire sociale des Algériens n'est certes pas un terrain vierge. Déjà dans les années 1960-1970, un certain nombre de travaux se sont intéressés aux appartenances sociales et professionnelles dans l'Algérie colonisée. Les sommes de Charles-André Julien et Charles-Robert Ageron font toujours référence, en particulier l'imposante étude de ce dernier sur les «Algériens musulmans $»^{10}$. Mais, en dehors des thèses d'André Nouschi sur les fellahs (paysans) du Constantinois ou de Gilbert Meynier sur la période 1900-191911, les recherches manquent pour apprécier par exemple le niveau de vie des populations ou la hiérarchie des salaires. Le monde du travail a d'abord été étudié sous l'angle des revendications syndicales et de la politisation des Algériens, souvent pour remonter le fil du nationalisme algérien dans une perspective d'histoire sociale du politique.

À partir des années 1970, Omar Carlier et Fanny Colonna, mais aussi les collègues et les étudiants algériens avec lesquels ils ont travaillé dans cette période très féconde, se sont employés à renouveler l'histoire sociale de l'Algérie colonisée. Leurs recherches ont aussi bien porté sur le travail ${ }^{12}$, que sur le terrain rural ${ }^{13}$ et le monde des citadins ${ }^{14}$; elles ont ainsi fait émerger des figures de médiateurs—salariés, militants, cafetiers, instituteurs—et de petits « intel- 
lectuels du certificat d'études ${ }^{15}$ qui tirent profit de leurs compétences scolaires pour subvenir aux besoins de leurs proches. En position charnière au sein de la population colonisée, ces hommes maîtrisent les codes et les attentes de l'État colonial. Pour autant, ces travaux sont ensuite restés relativement isolés dans une Algérie indépendante obnubilée par la "guerre de libération ». En France, le déclin de l'histoire sociale à partir des années 1980, l'ouverture des archives de la guerre d'indépendance dans les années 1990 et la difficulté pour les chercheurs étrangers de se rendre en Algérie pendant la guerre civile expliquent en partie le reflux de ces questionnements.

Les recherches manquent aussi pour écrire une synthèse de l'histoire sociale des migrants algériens, même si l'on peut s'appuyer sur quelques études particulièrement riches qui s'inscrivent dans l'histoire du mouvement ouvrier. Geneviève Massard-Guilbaud amende le modèle des « trois âges » de l'émigration d'Abdelmayek Sayad dans son travail sur les Algériens de Lyon dans $l^{\prime}$ entre-deux-guerres ${ }^{16}$, tandis que Laure Pitti met au jour la spécificité du traitement post-colonial des migrants algériens employés chez Renault des années 1950 à $1970^{17}$. Les trajectoires particulières des harkis au sein du groupe des migrants algériens ont également attiré l'attention des chercheurs ${ }^{18}$. $\mathrm{D}^{\prime}$ autres travaux ont privilégié l'échelle locale pour éclairer les parcours professionnels et familiaux des migrants algériens ${ }^{19}$, mais la plupart restent centrés sur les années de guerre et s'arrêtent en 1962, alors même que l'immigration algérienne en France s'est prolongée bien au-delà.

Ces études forment un corpus qui mérite donc encore d'être étoffé pour saisir l'évolution du groupe des Algériens au vingtième siècle. Depuis quelques années cependant, des thèses ont placé l'histoire sociale au centre de leurs questionnements ${ }^{20}$. Les travaux britanniques et américains, insuffisamment cités ici, participent à ce renouvellement prometteur de l'histoire de l'Algérie et des Algériens ${ }^{21}$.

\section{Écrire une histoire sociale à partir des archives de l'État?}

L'historien du social reste confronté à un problème majeur : appréhender des hommes et des femmes qui écrivent peu et qui sont minorés par les sources officielles $^{22}$. Peut-il écrire une histoire par le bas à partir d'une documentation en langue française majoritairement produite par l'administration coloniale et les élites locales ? Comment proposer une relecture critique des archives du pouvoir et comment surtout y déceler les traces des anonymes?

Les sources produites par les autorités coloniales sont imprégnées des logiques de pouvoir dont elles révèlent les priorités, parfois de manière caricaturale : quadriller le territoire colonisé, réduire les coûts, encadrer les flux migratoires ou surveiller les populations. Les archives des PTT racontent ainsi d'abord l'histoire d'employés citoyens, venus de métropole ou nés en Algérie. Dans cette nébuleuse administrative, le personnel algérien est beaucoup plus 
difficile à saisir, avant tout parce que la production écrite est nettement moins importante s'agissant des non-titulaires, voire inexistante pour les journaliers qui gravitent aux abords de l'administration ${ }^{23}$. En dépit de leurs silences et de leurs lacunes, ces archives administratives rendent compte d'une réalité sociale complexe et font émerger un groupe méconnu de postiers non-citoyens particulièrement présents dans les zones rurales (Annick Lacroix).

Les échanges entre les municipalités, les préfectures et les services administratifs méritent également d'être réexaminés, pour y pister de manière plus systématique les colonisés, visibles au détour d'une lettre ou d'une pétition. Éclairant l'échelon le plus local du millefeuille administratif, cette documentation dit quelque chose des rapports sociaux qui s'établissent dans l'Algérie colonisée et aident à complexifier les notions de " monde du contact » ou de « rencontre coloniale ${ }^{24}$. Particulièrement riches pour le vingtième siècle, les gisements de dossiers nominatifs et les recensements conservés dans les archives locales et nationales de France et d'Algérie méritent également d'être mis à profit pour esquisser une sociologie d'employés et d'usagers de l'administration coloniale et post-coloniale, ou encore pour faire l'histoire de certains quartiers et de leur évolution. En métropole, la forte surveillance policière à laquelle est soumise la population algérienne est à l'origine de sources abondantes qui permettent d'appréhender le quotidien des travailleurs, notamment celui des gérants de cafés, figures centrales de la communauté algérienne (Neil Macmaster).

La production bureaucratique de l'État offre donc des éléments concrets pour écrire une histoire sociale des Algériens, même s'il est indispensable de la confronter à des sources alternatives. La presse locale et les archives privées et familiales constituent des atouts précieux pour reconstituer des interactions ordinaires et des trajectoires individuelles ${ }^{25}$. La mobilisation d'archives syndicales peut encore enrichir le tableau. Mais surtout, les sources en arabe produites par les tribunaux religieux (cadi), les confréries (zaoü̈a), les assemblées villageoises (djemaas), ou plus largement par des familles ou des individus lettrés, restent sous-exploitées par les historiens français, alors qu'elles pourraient être mises au service d'une histoire sociale. Leurs potentialités sont encore à explorer, notamment pour cerner les mondes qui échappent à l'administration (Hannah-Louise Clark) $^{26}$. Reste que si ces sources écrites donnent enfin la parole aux colonisés, celle-ci est largement accaparée par les notables et les savants qui reflètent en partie seulement le point de vue des masses algériennes souvent illettrées. C'est pourquoi les rapports des manifestant(e)s du 17 octobre 1961 constituent une source exceptionnelle (Emmanuel Blanchard).

\section{Combiner les approches, diversifier les outils d'analyse}

Prolongeant un récent article d'Emmanuel Blanchard et Sylvie Thénault, qui appelait à « redonner toute leur place aux concepts et aux outils ordinaires 
[de l'] histoire sociale ${ }^{27}$ », ce dossier croise les approches et mobilise tour à tour des méthodes quantitatives et qualitatives, pour pister sans relâche les groupes $\mathrm{d}^{\prime}$ Algériens ${ }^{28}$. L'article d'Hannah-Louise Clark revient sur l'entreprise de vaccination contre la variole menée dans les campagnes algériennes durant la première moitié du vingtième siècle. L'intérêt pour les auxiliaires médicaux « indigènes », chargés localement de mettre en œuvre cette politique de santé, permet de pénétrer au cœur des communes mixtes et des douars. L'historienne interroge alors la production bureaucratique de ces acteurs rémunérés par les autorités coloniales. Dotés de compétences scripturaires, ils révèlent le quotidien des populations algériennes rurales qu'ils soignent.

Dans une perspective voisine, Annick Lacroix explore la position intermédiaire des postiers algériens, un autre groupe d'employés non-citoyens de l'État colonial. L'article décline les fonctions subalternes qu'ils occupent aux PTT, avant d'examiner la place de ces derniers par rapport à l'ensemble de la société colonisée, à partir du traitement statistique de dossiers nominatifs. La constitution d'une base de données permet d'estimer les ressources sociales, économiques ou scolaires de candidats à un emploi dans le Constantinois. Le dépouillement des dossiers puis le codage des variables apprivoisent la source et obligent à réfléchir à ses conditions de production. Pas vraiment membres d'une élite, mais déjà sortis de la masse des fellahs (paysans), ces postiers « indigènes » se placent en position d'observateurs privilégiés, entre l'État colonial et les usagers-administrés.

Deux articles renouvellent l'étude de la guerre d'indépendance en métropole et mobilisent une échelle micro (le café, la rue, le quartier) pour réévaluer la place des Algériens dans la capitale et aborder les stratégies des militants à différents niveaux de responsabilité. Largement fondée sur les archives de la préfecture de police, l'enquête de Neil Macmaster montre comment à Paris les solidarités familiales et les réseaux de la sociabilité et du commerce des migrants algériens ont servi d'appui à l'organisation clandestine du FLN (Front de libération nationale). Emmanuel Blanchard revient sur un événement maintenant connu de la guerre en métropole, en replaçant au centre les Algériens pour restituer leur vécu de la manifestation du 17 octobre 1961, ainsi que le sens qu'ils y attachent. Sa contribution met en relief la participation, trop souvent minorée, des femmes.

Enfin, réexaminant les conditions de production des statistiques migratoires, Muriel Cohen travaille, à l'inverse, d'abord à une échelle impériale, pour éclairer les circulations des Algériens entre Algérie et métropole du lendemain de la Seconde Guerre mondiale aux années 1980. Mais une fois décrits les cadres politiques dans lesquels se jouent ces mouvements, la suite de l'article révèle les pratiques circulatoires telles qu'elles sont décrites dans les récits des migrants. Le recours à la source orale—qui passe notamment par l'accès aux dialectes algériens ou des enquêtes sur le terrain ${ }^{29}$ - offre en effet des pistes pour saisir les trajectoires et le quotidien des populations dans la période postérieure aux années 1940. La démarche rappelle l'importance pour les chercheurs de diversifier et de combiner leurs outils d'analyse. 
L'écriture d'une histoire sociale des Algériens a donc tout à gagner à croiser les matériaux et les approches afin de tirer parti d'un dialogue fructueux entre les disciplines des sciences sociales. Pour s'affranchir des limites imposées par les archives coloniales, nous espérons montrer que le chercheur peut construire ses propres sources, en collectant des documents privés, notamment en langue arabe, en constituant des corpus sériels ou en réalisant des entretiens. Il peut en outre engager une démarche réflexive, déconstruire les catégories coloniales, ou simplement mieux donner à voir les paramètres de son enquête. L'articulation des échelles enfin doit être mise au service de cette histoire sociale des Algériens : elle implique de tenir ensemble les espaces algérien et métropolitain, d'élargir la perspective au Maghreb ou au monde musulman, mais aussi de porter l'attention aux continuités pré-coloniales et post-coloniales. Si ce dossier ne répond qu'en partie à ce cahier des charges, il montre déjà que derrière le regard étatique, il est possible de faire surgir les réalités locales et quotidiennes, au cœur d'un quartier parisien comme dans un douar de commune mixte. Du facteur à l'auxiliaire médical des campagnes algériennes, en passant par le gérant de café militant ou le touriste-migrant, certains mettent à profit ou contournent un dispositif colonial ou étatique pourtant pesant. Les Algériens ne sont dès lors ni un bloc monolithique, ni le réceptacle passif des politiques d'un État colonial-Léviathan, mais bien ces hommes et ces femmes, ruraux et citadins, fonctionnaires, migrants et militants dont on touche enfin du doigt la diversité et la vie quotidienne.

Muriel Cohen est enseignante en lycée, chercheuse associée au Centre d'histoire sociale du vingtième siècle, Université Paris 1 . Ses domaines de recherche sont l'histoire de l'immigration, l'histoire urbaine et l'histoire coloniale et post-coloniale. Elle a achevé en 2013 une thèse sur les politiques menées à l'égard des familles algériennes en France entre 1945 et 1985 et leurs trajectoires sociales et résidentielles. Ses articles portent sur les bidonvilles de Nanterre, les cités de transit, la politique de regroupement familial et l'histoire sociale des Algériens en France.

AnNick LaCroix est agrégée d'histoire, chercheuse associée à l'Institut des sciences sociales du politique à l'École normale supérieure de Cachan. Elle enseigne à l'Institut national des langues et civilisations orientales. Ses domaines de recherches portent sur l'histoire de l'État et des sociétés coloniales au Maghreb, et notamment sur l'histoire du travail. Elle a soutenu en 2014 une thèse sur l'administration des postes, télégraphes, téléphones en Algérie entre le milieu du dix-neuvième siècle et la Seconde Guerre mondiale. 


\section{Notes}

* Nous tenons à remercier pour leurs conseils et leur relecture Raphaëlle Branche, M'Hamed Oualdi et Emmanuel Szurek.

1. Raphaëlle Branche, La Torture et l'armée pendant la guerre d'Algérie : 1954-1962 (Paris : Gallimard, 2001); Sylvie Thénault, Une drôle de justice : Les magistrats dans la guerre d'Algérie (Paris : la Découverte, 2004); Didier Guignard, L'Abus de pouvoir dans l'Algérie coloniale, 1880-1914: Visibilité et singularité (Paris : Presses universitaires de Paris Ouest, 2010) ; Sylvie Thénault, Violence ordinaire dans l'Algérie coloniale : Camps, internements, assignations à résidence (Paris : Odile Jacob, 2011).

2. Peggy Derder, L'Immigration algérienne et les pouvoirs publics dans le département de la Seine (Paris : L'Harmattan, 2001) ; Linda Amiri, La Bataille de France, la guerre d'Algérie en métropole (Paris : Robert Laffont, 2004) ; Jim House et Neil Macmaster, Paris 1961 : Les Algériens, la terreur d'État et la mémoire (Paris : Tallandier, 2008); Emmanuel Blanchard, La Police parisienne et les Algériens (1944-1962) (Paris : Nouveau monde, 2011).

3. Celui-ci a largement été initié autour du séminaire organisé depuis 2008 par Emmanuel Blanchard et Sylvie Thénault, «Écrire une histoire sociale de l'Algérie colonisée » (CHS-Paris 1 / CESDIP-Saint-Quentin). Voir aussi Emmanuel Blanchard et Sylvie Thénault, éd., "La société du contact dans l'Algérie coloniale », Le Mouvement social 236, 3 (2011).

4. Isabelle Grangaud et M'hamed Oualdi, « Tout est-il colonial dans le Maghreb ? Ce que les travaux des historiens modernistes peuvent apporter ", L'Année du Maghreb 10 (2014) : 233-54.

5. Frederick Cooper et Ann Laura Stoler, eds., Tensions of Empire : Colonial Cultures in a Bourgeois World (Berkeley : University of California Press, 1997), 1-58.

6. Voir notamment le colloque "Histoire de l'Algérie coloniale : longue durée et désenclavement », organisé par Hélène Blais, Claire Fredj et Sylvie Thénault (18 et 19 avril 2013 à l’Université Paris Ouest-Nanterre-La Défense).

7. Raphaëlle Branche, La Guerre d'Algérie : Une histoire apaisée ? (Paris : Seuil, 2005), 349.

8. On sous-estime parfois les crispations que suscite encore aujourd'hui en Algérie l'usage du terme "indigène ». Daho Djerbal, "Une violence coutumière », postface aux textes sur la torture, Denise et Robert Barrat, Algérie, 1956 : Livre blanc sur la répression (Alger : Barzakh, 2001).

9. Alain Girard et Joseph Leriche, dir., Les Algériens en France : Étude démographique et sociale (Paris : PUF-INED, 1955).

10. Charles-André Julien, Histoire de l'Algérie contemporaine : La conquête et les débuts de la colonisation, 1827-1871 (Paris : PUF, 1986) ; Charles-Robert Ageron, Histoire de l'Algérie contemporaine : De l'insurrection de 1871 au déclenchement de la guerre de libération, 1954 (Paris : PUF, 1979) ; Charles-Robert Ageron, Les Algériens musulmans et la France (1871-1919) (Paris : PUF, 1968).

11. André Nouschi, Enquête sur le niveau de vie des populations rurales constantinoises, de la conquête jusqu'en 1919 : Essai d'histoire économique et sociale (Paris : PUF, 1961) ; Gilbert Meynier, L'Algérie révélée : La guerre de 1914-1918 et le premier quart du XXe siècle (Genève : Droz, 1981).

12. Omar Carlier, «Les traminots algérois des années $1930:$ un groupe social médiateur et novateur », Le Mouvement social 146 (mars 1989) : 61-89; Fanny Colonna, Instituteurs algériens (1883-1939) (Paris : Presses de la Fondation nationale des sciences politiques, 1975).

13. Fanny Colonna, Le Meunier, les moines et le bandit: Des vies quotidiennes dans l'Aurès, Algérie, du XXe siècle, récits (Arles : Sindbad, 2009); Fanny Colonna, Savants paysans : 
Éléments d'histoire sociale sur l'Algérie rurale (Alger : Office des publications universitaires, 1987).

14. Omar Carlier, "Le café maure : sociabilité masculine et effervescence citoyenne (Algérie XVIIe-XXe siècles) », Annales. Économies, Sociétés, Civilisations 45, 4 (1990) : 975-1003.

15. Omar Carlier, «L'Espace et le Temps dans la recomposition du lien social : l'Algérie de 1830 à 1930 », in Urbanité arabe : Hommage à Bernard Lepetit, dir. Jocelyne Dakhlia (Arles : Actes Sud/Sinbad, 1998), 212.

16. Geneviève Massard-Guilbaud, Des Algériens à Lyon : De la Grande guerre au Front populaire (Paris : CIEMI, 1995).

17. Laure Pitti, "Ouvriers algériens à Renault-Billancourt, de la guerre d'Algérie aux grèves d'OS des années 1970 : Contribution à l'histoire sociale et politique des ouvriers étrangers en France » (Thèse de doctorat d'histoire, sous la direction de René Gallissot, Paris 8, Saint-Denis, 2002).

18. Voir notamment sur ces questions les travaux de Giulia Fabbiano et de Fatima Besnaci-Lancou.

19. Jean-René Genty, L'Immigration algérienne dans le Nord-Pas-de-Calais, 1909-1962 (Paris : L'Harmattan, 1999) ; Yves Frey, La Guerre d'Algérie en Alsace : Enquête sur les combattants de l'ombre, 1945-1965 (Strasbourg : La Nuée Bleue, 2013).

20. Marc André, « Des Algériennes à Lyon, 1947-1974 » (Thèse de doctorat, Université Paris IV-Sorbonne, Paris, 2014) ; Muriel Cohen, « Des familles invisibles : Politiques publiques et trajectoires résidentielles de l'immigration algérienne (1945-1985) » (Thèse de doctorat, Paris 1 Panthéon-Sorbonne, Paris, 2013) ; Annick Lacroix, « Une histoire sociale et spatiale de l'État dans l'Algérie colonisée : L'administration des postes, télégraphes et téléphones du milieu du XIXe siècle à la Seconde Guerre mondiale » (Thèse de doctorat, sous la direction de Raphaëlle Branche et Olivier Wievorka, École normale supérieure de Cachan, 2014).

21. Parmi de très nombreuses autres références, on peut citer : Julia Ann Clancy-Smith, Mediterraneans : North Africa and Europe in an Age of Migration, c. 1800-1900 (Berkeley : University of California Press, 2011) ; James Mc Dougall et Judith Scheele, eds., Saharan Frontiers : Space and Mobility in Northwest Africa (Bloomington : Indiana University Press, 2012) ; Amelia H. Lyons, The Civilizing Mission in the Metropole : Algerian Families and the French Welfare State During Decolonization (Stanford, CA : Stanford University Press, 2013) ; Hannah-Louise Clark, « Doctoring the Bled : Medical Auxiliaries, Therapeutic Modernities, and the Administration of Rural Life in Colonial Algeria, 1904-1962 »(PhD dissertation, Princeton University, 2014); Natalya Vince, Our Fighting Sisters : Nation, Memory and Gender in Algeria, 1954-2012 (Manchester : Manchester University Press, 2015).

22. Gayatri Spivak, "Can the Subaltern Speak? ", in Marxism and the Interpretation of Culture, ed. Cary Nelson and Lawrence Grossberg (Urbana : University of Illinois, 1988), 271-313; Emmanuelle Sibeud, « Post-Colonial et Colonial Studies : enjeux et débats ", Revue d'histoire moderne et contemporaine 51, 4-5 (2004) : 87-95 ; Jacques Pouchepadass, "Les Subaltern Studies ou la critique postcoloniale de la modernité », L'Homme, Revue française d'anthropologie 156 (janvier 2000) : 161-86.

23. Didier Guignard, Akihito Kudo et Raëd Bader, "Des lieux pour la recherche en Algérie », Bulletin de l'IHTP 83 (2004) : 158-68 ; Annick Lacroix, Claire Marynower et Hugo Vermeren, "Carnet de terrain : Retour sur les archives algériennes ", Vingtième Siècle, Revue d'histoire 110, 2 (2011) : 147-53. Perçus comme techniques et peu stratégiques, les archives des PTT conservées en Algérie sont ainsi restées près de cinquante ans à l'écart des regards historiens, au centre des archives nationales algériennes (Birkhadem), à la wilaya d'Alger ou dans certains bâtiments administratifs encore en activité (grande poste de Constantine). 
24. Jocelyne Dakhlia, «1830, une rencontre ? », in Histoire de l'Algérie à la période coloniale (1830-1962), dir. Abderrahmane Bouchene, Jean-Pierre Peyroulou, Ounassa Siari-Tengour et Sylvie Thénault (Paris : La Découverte, 2012) : 142-49. La notion de «monde du contact » est mobilisée par Annie Rey-Goldzeiguer, Aux origines de la guerre d'Algérie (1940-1945) : De Mers-el-Kébir aux massacres du nord-constantinois (Alger : Casbah Editions, 2002).

25. En l'absence de sources administratives, les archives de Monique Hervo, travailleuse sociale au bidonville de La Folie à Nanterre entre 1959 et 1971, déposées à l'Institut d'histoire du temps présent, constituent la principale source sur la vie quotidienne dans les bidonvilles.

26. Ismaill Warscheid, "Comment écrire un passé qui ne soit ni colonial, ni classique. Le cas du Tuwāt algérien ", in L'Orient créé par l'Orient, dir. François Pouillon et JeanClaude Vatin (Paris : Karthala, 2011), 213-27 ; Augustin Jomier, « Islâh ibâdite et intégration nationale : vers une communauté mozabite ? (1925-1964) », Revue des mondes musulmans et de la Méditerranée 132 (2012) : 175-95 ; Isabelle Grangaud, "Masking and Unmasking the Historic Quarters of Algiers. The Reassessment of an Archive ", in Walls of Algiers : Narratives of the City Through Text and Image, ed. Zeynep Celik, Julia Clancy-Smith et Frances Terpak (Los Angeles-Seattle and London : The Getty Research Institute, University of Washington Press), 179-97.

27. Emmanuel Blanchard et Sylvie Thénault, "Quel "monde du contact " ? Pour une histoire sociale de l'Algérie pendant la période coloniale ", Le Mouvement social 236, 3 (2011), 6.

28. Claire Lemercier, Carine Ollivier et Claire Zalc, « Articuler les approches qualitatives et quantitatives : Plaidoyer pour un bricolage raisonné », in Devenir chercheur : Écrire une thèse en sciences sociales, dir. Moritz Hunsmann et Sébastien Kapp (Paris : Éditions de l'EHESS, 2013), 125-43.

29. Voir les entretiens filmés avec d'anciens habitants des bidonvilles de Nanterre conservés à la BDIC : " Mémoire algérienne de l'immigration Oued Souf »; et aussi les travaux de Natalya Vince, Malika Rahal et Andrea Brazzoduro. Par exemple, Andrea Brazzoduro, Soldati senza causa : Memorie della guerra d'Algeria (Roma-Bari, Italie : Laterza, 2012). 